\title{
Internet of things and the human body
}

Volume 5 Issue 2 - 2017

\section{Short communication}

This is a short commentary considering the analogy which can be drawn between the current, "macro" Internet of Things (IoT) based applications (such as Smart Buildings, Smart Cities, Smart Car, etc.) and the future, highly probable, fusion between the "Body Sensor Networks" "and everything now covered by the nanomedicine domain. The aim is to offer "food for thought", questions which might not have yet concrete and full answers; performing such an exercise, between two areas - which are so different - is to understand where we are now and where (moreover, how), we can move forward, by exploring and exploiting the resources associated with human body.

The topic is not a brand new one; various communication channels already got it into the news. ${ }^{1,2}$

Smart "Everything" is this a common jargon; "everything" can be: phone, car, house, building, city, cloths, etc. Let's pick up on a "smart house"; smart sensors will measure various parameters, such as:
a. Quality of the Air
b. Level of Humidity (including possible water leaks)
c. Level of light
d. Temperature in various locations, including outside
e. Energy consumption (smart metering)
f. Homan presence

And will communicate to a central unit. Primary processing of the data will be done locally and actions will be triggered to adjust various parameters; some data will be transmitted to the cloud, for further processing. The whole process has a couple of objectives: ensuring the right level of comfort, safety and security of habitants and assets, quantifying and optimizing the energy consumption, etc. Who are the beneficiaries, besides the habitants? All service provides (water, electricity, gas, insurance, security, etc.). Processed data will help home owners to monitor what is going on in the house, to make it safer and more comfortable in terms of environment; will help service providers to ensure that the level of their services is right.

Let us move swiftly to human body. Which could be the ingredients for building a "Smart Body", in analogy with the "Smart House"? There are some evident benefits for developing and implementing such architecture: a "condition-based", personalized medical care, a better use of medical resources, faster and personalized interventions and not the least, personalized healthcare insurance plans, etc.

The answer can be, apparently, very simple:

a. Nano- objects collecting data about the human body and acting for adjusting some of the human body physiological parameters (Data Acquisition)

b. A communication path (Data Transmission); could be this represented by what is known and defined as "Body Sensors/Area Networks" 3,4

\author{
Octavian Buiu, Bogdan Catalin Serban, \\ Octavian lonescu \\ National R\&D Institute for Microtechnologies, Romania
}

Correspondence: Octavian Buiu, National R\&D Institute for Microtechnologies, Bucharest, Romania

Email Octavian.buiu@gmail.com

Received: February 19, 2017| Published: March 17, 2017

c. A Local Data Processing unit and further on, a connection to the CLOUD (for further Data Processing and Decision Taking)

Let's have a look at the nanomedicine first, as a research area; what does it mean? Could this really be the first ingredient? If we start with the British Society for Nanomedicine. ${ }^{5}$ their definition will point out that "is simply the application of nanotechnologies in a healthcare setting". ${ }^{6}$ will tight the nanomedicine to the wider domain of medicine ("Nanomedicine is a branch of medicine that applies the knowledge and tools of nanotechnology to the prevention and treatment of disease."), while. ${ }^{7}$ does assign the domain in the field of nanotechnology applications and keeps the definition quite wide - "Nanomedicine ranges from the medical applications of nanomaterials and biological devices, to nanoelectronic biosensors, and even possible future applications of molecular nanotechnology such as biological machines" (Figure 1).

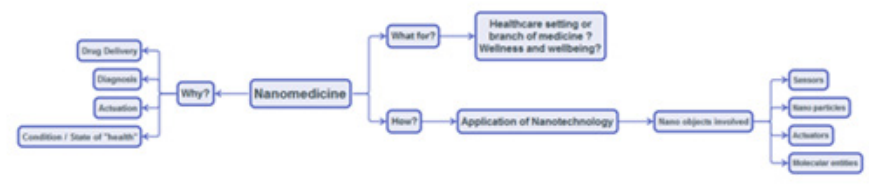

Figure I Blow does summarize the coverage given by the definitions discussed above.

After being discussed at large, at the conceptual level [8,9], Body Sensors/Area Networks is nowadays (more and more) used in practical applications. This was made possible by the advancements in the field of wearable sensors, as well as short-distance communication protocols and their hardware implementation. This day, a wide range of applications covering mostly what is known as "Wellness and wellbeing" are commercially available. Challenges are still there: new sensors, more energy efficient, coupled with harvesting devices; and all being able to communicate, in a reliable and secure way with a "central unit" kind of device (smart-phone, smart watch, etc.). A special field is represented by the implanted wireless sensors, such as those used in total knee arthroplasty. ${ }^{10}$ or in prosthetic limb (such as LINX. $\left.{ }^{11}\right)$.

But here we have another challenge (in the context of the current commentary); can the nano-objects (as means in use by the 
nanomedicine field) be integrated by these networks? How to connect, for example, a drug delivery process and its effects with recordings made by the current set of wearable sensors?

This brings us to the last of the three ingredients, i. e., Data Processing (local and central). Where and how we are placing these two functions? Of course, some processing can be done at the level of Body Sensors/Area Networks; challenge is given by the capacity of the current available hardware, especially if more of the implanted sensors and nano-robts with communication capabilities will be further included.

How we move to the central Data Processing? Of course, various smart devices - collecting information from wearables - are directly connected to the cloud processing capabilities. But can we implement and perform more processing at the human body level? Can we somehow - transfer the data collected about our body to the brain for processing? How this interface should look and behave like? Can augmented reality (AR) play a role? Widely used by medical staff to monitor various medical processes, could it be the solution to be used now by the patients to monitor and understand what is happening in and with their body (Figure 2)?

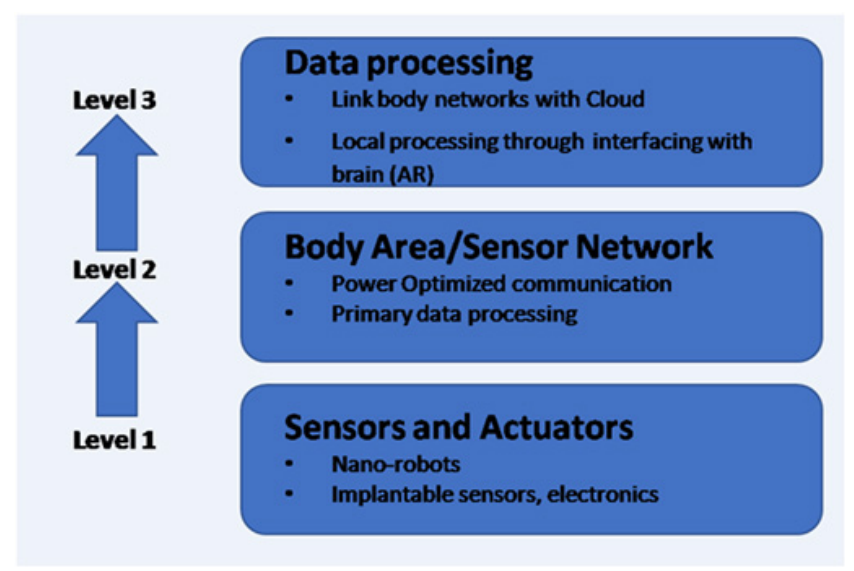

Figure 2 Does summarize - in a graphical manner - A layered structure, showing the flow of information "bottom-up" (from level (I) to level (3)). Can we imagine this flow also working in reverse (level 3 to level I)? Food for thought!

\section{Acknowledgments}

None.

\section{Conflicts of interest}

None.

\section{References}

1. Courtney Olean Paige From the Internet of Things to the Internet of the Body. 2014

2. John Brandon The Internet of Things... for the Human Body? 2017

3. Prabha Sundaravadivel, Saraju P Mohanty, Elias Kougianos et al. Exploring Human Body Communications for IoT Enabled Ambulatory Health Monitoring Systems. IEEE International Symposium on Nanoelectronic and Information Systems (iNIS), India. 2016

4. Moeen Hassanalieragh, Alex Page, Tolga Soyata, et al. Health Monitoring and Management Using Internet-of-Things (IoT) Sensing with Cloudbased Processing: Opportunities and Challenges. IEEE International Conference on Services Computing, India, pp. 2015;285-292.

5. British Society for Nanomedicine.

6. Nanomedicine, Nature, USA.

7. Nanomedicine.

8. Xiaochen Lai, Quanli Liu, Xin Wei et al. A Survey of Body Sensor Networks. Sensors. 2013;13(5):5406-5447.

9. Douglas McIlwraith, Guang-Zhong Yang Body Sensor Networks for Sport, Wellbeing and Health. Sensor Networks, pp. 2009;349-381.

10. OrthoSensor VERASENSE Overview: Benefits of Sensor-Assisted Knee Replacement Surgery.

11. LiNX. 